\title{
LA DOMESTICACIÓN DEL PAISAJE EN ENCLAVES PLURICULTURALES DEL SUR DE Misiones (ARgentina): UnA APROXIMACIÓN A TRAVÉs DE LOS CÍTRICOS
}

\author{
PABLO CÉSAR STAMPELLA
}

\begin{abstract}
Resumen: Los cítricos son frutales introducidos tempranamente a América. Actualmente para Misiones se reconocen 30 etnovariedades (9 etnoespecies), cultivadas de maneras diversas. En la presente contribución se describen los microambientes donde se encuentran, las prácticas sobre dichos microambientes y las etnovariedades presentes en cada uno para luego analizar, desde un enfoque relacional, el efecto de las prácticas de manejo en los procesos de domesticación de las plantas y del paisaje. Los trabajos de campo fueron realizados en Teyú Cuaré (San Ignacio) y Cerro Mártires (Concepción), provincia de Misiones (Argentina), durante los años 2010 a 2013. Se realizaron entrevistas abiertas y semiestructuradas, y caminatas con los informantes. Se relevaron 7 microambientes definidos en relación a la presencia de cítricos, a las prácticas de manejo focalizadas en las plantas propiamente dichas como también sobre los microambientes o grupos de microambientes, así como por aspectos relativos a la vegetación, suelo, disponibilidad hídrica y cercanía al hogar. La diversidad de cítricos registrada representa un continuum de variedades representantes del proceso de domesticación en diferentes sentidos, coincidente a su vez con las prácticas de manejo diversificadoras. El estudio de las prácticas de manejo permite observar patrones de manejo actuales como también interpretar los paisajes actuales infiriendo las prácticas del pasado.
\end{abstract}

Palabras clave: Criollos, Diversificación, Domesticación del paisaje, Etnobotánica.

Summary: Domestication of the landscape in pluricultural enclaves of South of Misiones (Argentina): an approach through the citrus. Citrus are fruit trees early introduced into America. Nowadays in Misiones there are 30 ethnovarieties ( 9 ethnospecies) locally recognized and cultivated in different ways. The objectives of this contribution are to describe the microenvironments where they grow, the practices on those microenvironments and the ethnovarieties present in each one of them, and then to analyze, from a relational approach, the effect of management practices on the processes of domestication of plants and landscape. Fieldwork was performed in Teyu Cuare (San Ignacio) and Cerro Martires (Concepción), province of Misiones (Argentina), during the years 2010 to 2013. Open-ended and semi-structured interviews were carried out as well as walks with the informants. Seven microenvironments were determined in relation to the presence of citrus, management practices on the plants themselves as well as on microenvironments or groups of microenvironments and by aspects related to vegetation, soil, water availability and proximity to the home. The diversity of citrus recorded in the different microenvironments represents a continuum of varieties representative of the process of domestication in different senses, coinciding in turn with the diversification management practices. The study of management practices allows us to observe current management patterns as well as interpreting nowadays landscapes by inferring past practices.

Key words: Criollos, Diversification, Landscape domestication, Ethnobotany.

\footnotetext{
1 Laboratorio de Etnobotánica y Botánica Aplicada (LEBA), Facultad de Ciencias Naturales y Museo (FCNyM), Universidad Nacional de La Plata (UNLP), Calle 64 n³, CP: 1900, La Plata, Argentina. Becario Postdoctoral, Consejo Nacional de Investigaciones Científicas y Técnicas (CONICET). E-mail: pstampella@yahoo.com
} 


\section{INTRODUCCIÓN}

Los frutales cítricos fueron introducidos a América a partir del siglo XVI desde diversos enclaves y por diferentes rutas de ingreso. Una vez introducidos devinieron en elementos conspicuos del ambiente siendo empleados con diversos fines, incluso en la planificación de los paisajes por comunidades locales (Stampella et al., 2013b; Riat et al., 2015). En el sur de Misiones, se han relevado 30 etnovariedades correspondientes a 9 etnoespecies de cítricos, varias de ellas espontáneas en las selvas con baja a media intervención antrópica (Stampella, 2016). Esta modificación de los ecosistemas por las sociedades data de varios siglos antes del contacto entre el Viejo y el Nuevo Mundo, tal como lo destaca la Ecología Histórica (Balée, 1998), al no considerar la existencia de ecosistemas prístinos, exentos de prácticas humanas. La historia local de cinco siglos, la presencia de variedades espontáneas y la amplia variabilidad de usos de estos frutales refleja procesos de apropiación y resignificación por las comunidades locales como "plantas del monte", rompiendo a su vez la dicotomía nativo/ exótico (Pochettino et al., 2015).

Estos enclaves locales se basan en la Estrategia de Uso Múltiple (EUM) de los bienes comunes aplicada desde la Unidad Doméstica (UD) al nivel de especies, unidades ecogeográficas y paisajes, con fines de subsistencia y de intercambio económico (Toledo et al., 2007; Riat et al., 2015). Además están caracterizados por el bajo $-\mathrm{o}$ incluso nulo- empleo de insumos externos (agrotóxicos, fertilizantes) y criterios de apropiación cuyos intercambios ecológicos son tan importantes como los económicos. Esto lleva a que se consideren como reservorios dinámicos de Conocimientos Ecológicos Locales (Toledo \& Bassols, 2008) constantemente puestos en juego mediante diversas prácticas dirigidas a las plantas y el paisaje, que resultan fundamentales conocer para el implemento de estrategias de conservación in situ de tales bienes.

En relación a los procesos locales de selección cultural, la diversidad de prácticas sobre una misma especie permite la aparición y conservación dinámica (in situ) de las etnovariedades generadas. Estos procesos han sido abordados desde diferentes ópticas. En un trabajo previo (Stampella, 2016) se relacionó la diversidad de cítricos espontáneos y cultivados con la riqueza de percepciones de la variabilidad, la coexistencia de diferentes prácticas de manejo y el análisis de las prácticas del pasado. Este espectro de prácticas locales sobre el entorno puede ser abordado desde los diferentes grados de relación ser humano-planta o ser humanoambiente que abarcan la recolección de frutos y consumo in situ de los mismos, manejo de parches de vegetación, cultivo de plantas en diferentes situaciones de cuidado, y domesticación completa de las mismas, proceso logrado por manipulación del genotipo vegetal (Casas, 2001; Lema, 2010).

De esta manera, las prácticas de manejo, junto a las características geográficas intrínsecas (tipo de vegetación dominante, topografía, suelos, disponibilidad hídrica) configuran distintos microambientes o zonas ecológicas (Martin, 2001; Guarino \& Hoogendijk, 2004) caracterizados localmente por la presencia de los cítricos. Estos microambientes son condicionados por las prácticas de manejo que los transforman unos en otros, recreándolos y derivando en procesos de domesticación de genotipos de plantas y del paisaje (Balée, 1998; Capparelli et al., 2011).

El proceso de domesticación es bien conocido en las plantas anuales pero en cambio ha sido abordado más recientemente para el caso de las perennes ya que constituyen un modelo más abierto debido a la presencia de fases juveniles extensas, polinización cruzada, altas tasas de flujo génico (intra e interespecíficos) y diversos mecanismos de reproducción sexual y asexual (Zohary, 2004; Miller \& Gross, 2011). Al igual que en las plantas anuales, la selección cultural en perennes puede resultar en diferentes procesos de domesticación -o no- visualizándose incluso los matices de dichos procesos. En la bibliografía abundan los ejemplos acerca del cultivo de plantas arbóreas en las que se evidencian ciertos cambios morfológicos y organolépticos en determinados órganos vegetales sin que ocurra domesticación: Adansonia spp. (Malvaceae), Faidherbia albida (Delile) A.Chev. (Fabaceae), Butyrospermum sp. (Arecaceae) y Elaeis guineensis Jacq. (Arecaceae) (Harlan, 1992). En cambio, en la "domesticación incipiente" las prácticas de manejo no están dirigidas hacia la dependencia reproductiva de la planta sino a generar cambios morfológicos-organolépticos de acuerdo a diferentes criterios, como ocurre en: Leucaena esculenta (DC.) Benth. (Fabaceae), Blighia sapida 
K.D. Koenig (Sapindaceae), Sideroxylon palmeri (Rose) T.D. Penn. (Sapotaceae), Crescentia cujete L. (Bignoniaceae) y Spondias tuberosa Arruda (Anacardiaceae) (Casas \& Caballero, 1996; González-Soberanis \& Casas, 2004; Marius et al., 2010; Aguirre-Dugua et al., 2012; Lins Neto et al., 2012).

Estas prácticas diversificadoras sobre las poblaciones de plantas espontáneas pueden resumirse en el "modelo de silvicultura" planteado por Casas (2001), donde se destacan los criterios diferenciales de selección cultural que determinan aquellas plantas espontáneas (o fenotipos) que serán erradicadas o cultivadas en un sentido amplio. Entre estas últimas incluso se distinguen diferentes grados en la relación seres humanos-plantas: plantas preferidas para la recolección, que quedarán en pie (toleradas), que serán favorecidas (fomentadas) y/o aquellas que serán cultivadas en sentido estricto (protegidas). Cabe destacar que, a diferencia de las plantas anuales, la selección cultural no es realizada entre la cosecha y la siembra sino que opera seleccionando (erradicando o tolerando, y muchas veces fomentando y/o protegiendo) la variabilidad generada natural/culturalmente por diferentes vías, de manera consciente e inconsciente (Emperaire et al., 2001).
El objetivo del presente trabajo es describir los distintos microambientes definidos en relación a la presencia de cítricos, las prácticas sobre los mismos y las etnovariedades presentes en cada uno, como procesos que contribuyen a entender los mecanismos locales involucrados en la generación de la variabilidad de Citrus en el sur de Misiones. Se discuten las prácticas de manejo sobre los distintos microambientes de acuerdo a la propuesta relacional de Casas (2001).

\section{Materiales y Métodos}

\section{Área de estudio}

Los trabajos de campo fueron llevados a cabo en los parajes Teyú Cuaré y Cerro Mártires (Tabla 1; Figura 1). Estos enclaves están ubicados en el área de transición entre los distritos de las Selvas Mixtas y los Campos de la provincia Fitogeográfica Paranense (Cabrera, 1976), observándose una dinámica particular entre estas dos unidades de vegetación, conformada por comunidades xerofíticas asociadas a afloramientos rocosos que Martínez-Crovetto (1963) describe como distrito del Urunday (Astronium balansae Engl., Anacardiaceae).

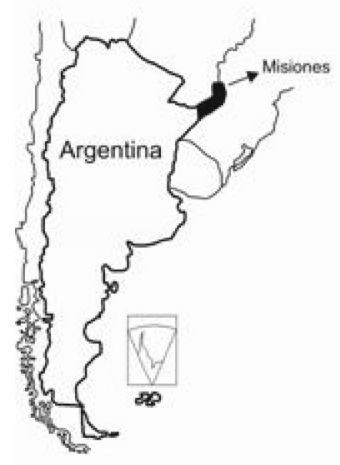

Sitio de estudio

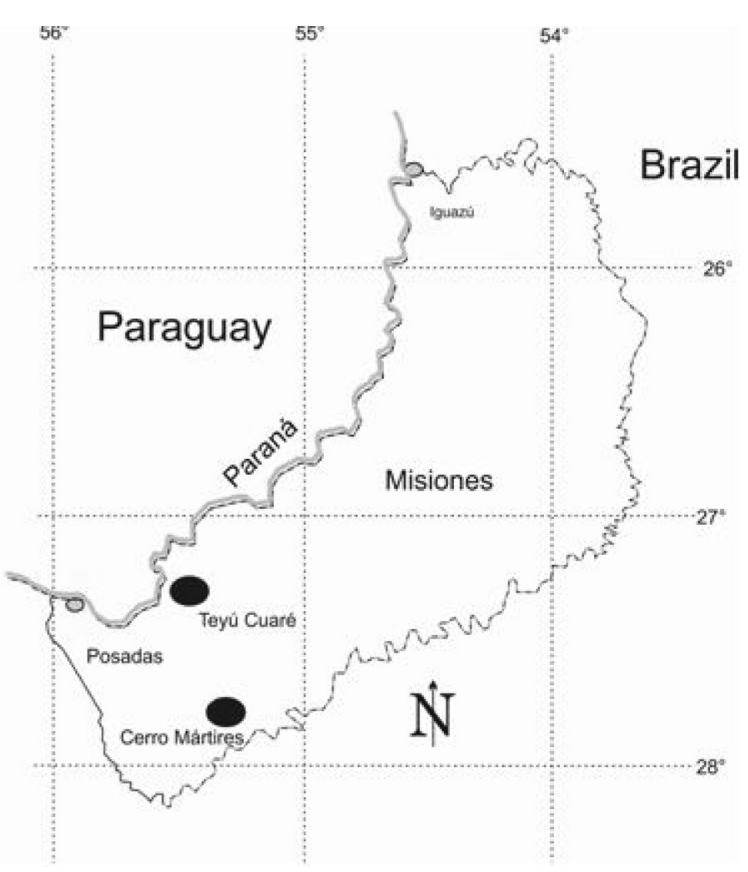

Fig. 1. Área de estudio. 
Tabla 1. Enclaves estudiados discriminados por cuenca, ubicación geográfica, distritos fitogeográficos principales y UD e informantes consultados.

\begin{tabular}{|c|c|c|}
\hline Cuenca & Paraná & Uruguay \\
\hline Departamento & San Ignacio & $\begin{array}{l}\text { Concepción } \\
\text { de la Sierra }\end{array}$ \\
\hline Municipio & San Ignacio & Santa María \\
\hline Localidad & Teyú Cuaré & Cerro Mártires \\
\hline $\begin{array}{l}\text { Distritos } \\
\text { fitogeográficos }\end{array}$ & $\begin{array}{c}\text { Fluvial } \\
\text { Paranaense / } \\
\text { del Urunday }\end{array}$ & $\begin{array}{c}\text { del urunday / de } \\
\text { los Laureles }\end{array}$ \\
\hline $\begin{array}{l}\text { Coordenadas } \\
\text { geográficas }\end{array}$ & $\begin{array}{l}27^{\circ} 17^{\prime} 55^{\prime \prime} \mathrm{S} \\
55^{\circ} 34^{\prime} 27^{\prime \prime} \mathrm{W}\end{array}$ & $\begin{array}{l}27^{\circ} 49^{\prime} 11^{\prime \prime} \mathrm{S} \\
55^{\circ} 24^{\prime} 41^{\prime \prime} \mathrm{W}\end{array}$ \\
\hline UD & 6 & 12 \\
\hline Informantes & 6 & 19 \\
\hline
\end{tabular}

Esta provincia presenta altos índices de diversidad biológica y cultural (Belastegui, 2004; Placci \& Di Bitetti, 2006), reflejado en la flora, fauna y mosaico cultural producto de 5 siglos de historia. Así, los ambientes hallados por los inmigrantes fueron transformados en sistemas agrícolas, forestales y agrosilvícolas con distinto grado de diversificación (Chebez \& Hilgert, 2003). Las actividades productivas dominantes en la zona son la silvicultura (especialmente pino y eucalipto), la cría de ganado vacuno y plantaciones de yerba mate, té, tabaco y cítricos, actividades que proporcionan empleos temporarios (changas, jornales) a los pobladores locales (Izquierdo et al., 2010).

Las UD estudiadas están conformadas por familias extendidas de "criollos", definidos como la fusión de los pueblos originarios con diferentes vertientes de inmigrantes en época colonial y de principios de siglo XX, con ciudadanos brasileños y paraguayos (Belastegui, 2006). Asimismo, fueron visitadas algunas familias descendientes de inmigrantes polacos y ucranianos, denominados localmente colonos. Las comunidades M'Bya (paisanos) no fueron visitadas aunque algunos de sus miembros formaban parte permanente o temporal de algunas UD. En el sur de la provincia pueden distinguirse 2 cuencas separadas por las serranías centrales y asociadas a los dos países limítrofes (Paraguay y Brasil) al otro lado de los grandes ríos.

Las familias se apropian del ambiente mediante diversas prácticas productivas como también la caza, pesca y recolección de frutos de la selva. Las parcelas de cultivo varían en diversidad de plantas toleradas y cultivadas, y son establecidas mediante roza y quema, sembrando generalmente diversas variedades de maíz (Zea mays L., Poaceae), mandioca (Manihot esculenta Crantz, Euphorbiaceae), batata [Ipomoea batatas (L.) Poir., Convolvulaceae], caña de azúcar (Saccharum officinarum L., Poaceae) y otros cultivos anuales como tabaco, soja, arroz y maní (Arachis hipogaea L., Fabaceae) (Schaden, 1998), que se intercalan con frutales perennes tolerados.

\section{Metodología}

Los trabajos de campo fueron realizados en diversas campañas entre los años 2010 y 2013, donde se convivió en la comunidad con algunas familias. Previamente se relevaron los microambientes reconocidos localmente donde crecen o son cultivados los cítricos. Para ello se indagó en los descriptores empleados para caracterizar los microambientes y su variabilidad. Posteriormente, se realizaron entrevistas abiertas y semiestructuradas y caminatas con los informantes por los distintos microambientes de la UD, complementadas con observación participante (Etkin \& Ticktin, 2010; Albuquerque et al., 2014). Preferentemente se trabajó con pobladores que mantienen contacto cotidiano a través del manejo de este tipo de bienes comunes, cuya edad fue muy variable (entre 18 y 88 años). Durante las entrevistas y caminatas a los distintos espacios se indagó acerca de la variabilidad cultivada (cítricos y otros frutales, como también otras plantas útiles), las prácticas sobre los mismos y las plantas, como también otros caracteres que definen los microambientes.

El material de herbario y los frutos colectados en este relevamiento fueron procesados, identificados y depositados en el Herbario de Plantas Útiles y en la Colección de Frutos y Semillas del Laboratorio de Etnobotánica y Botánica Aplicada (CFS, LEBA). Para su determinación se empleó bibliografía específica de cítricos (Swingle, 1943; Webber, 1943; Palacios, 1978; Anderson, 1996) y para la presentación de los nombres científicos se empleó The Plant List (2016), excepto para el género Citrus que se emplearon los 


\section{P. C. Stampella - Domesticación del paisaje en Misiones (Argentina)}

trabajos específicos de Mabberley $(1997,2004)$ y Zhang \& Mabberley (2008).

\section{Resultados}

\section{Microambientes}

Se relevaron 7 microambientes definidos en relación a la presencia de cítricos y complementados con las prácticas de manejo distintivas y aspectos relativos a la vegetación, disponibilidad hídrica y cercanía al hogar: "jardin", "monte frutal", "huerta", "campo", "rozado", "capuera" y "monte". De éstos sólo los tres primeros poseen riego controlado (Tabla 2). A continuación se describen las características de estos microambientes:

JARDÍN

Está ubicado alrededor del hogar y posee dimensiones variables. Generalmente está conformado por plantas cultivadas ornamentales, de sombra, frutales, condimenticias y medicinales, varias de ellas de valor emotivo. Parte de los frutales y de las "maderas de valor" (especies forestales) son tolerados durante las tareas de desmonte practicadas al construir la vivienda y el "jardín". Los cítricos hallados aquí corresponden a diversas variedades "injertadas" y "comunes".

\section{MONTE FRUTAL}

Es un espacio conformado por árboles y arbustos frutales que muchas veces se considera parte del "jardín" o de la "huerta" ("huerta de frutales"). $\mathrm{Su}$ composición es variable para cada UD pudiendo ser desde uniespecíficos ("naranjales", "bananales"), hasta diferentes combinaciones de especies (poliespecíficos). Mientras los primeros se asocian más a productos destinados a la venta en un mercado local, los otros son empleados para consumo de la UD y eventuales trueques. En

Tabla 2. Microambientes relevados indicando las prácticas sobre los mismos, la vegetación predominante, las etnovariedades de Citrus presentes y el grupo de etnovariedades al que pertenecen. Referencias: Las variedades comunes son aquellas que no participan en el injerto, propagadas mediante semillas; las de injerto son aquellas que intervienen en el injerto como portainjertos, propagadas de la misma manera que las comunes; y las injertadas son aquellas injertadas en sentido estricto sobre un portainjerto. 1. Cidra; Pomelos: 2. blanco, 3. rosado, 4. Cidra; Apepú: 5. de monte, 6. casera, 7. dulce; Naranja: 8. de monte, 9. de monte de fruto grande, 10. casera, 11. injertada, 12. de ombligo, 13. que guía; Mandarina: 14 . de monte, 15. casera, 16. injertada, 17. japonesa, 18. colorada, 19. injerto con apepú, 20. bergamota; Limón: 21. mandarina, 22. amarillo, 23. lima, 24. cidra, 25. sutil, 26. real; 27. Lima dulce; Quinoto: 28. común, 29. dulce; 30. Trifoliata.

\begin{tabular}{|c|c|c|c|c|}
\hline Microambiente & Etnovariedades & $\begin{array}{c}\text { Grupos de } \\
\text { Etnovariedades }\end{array}$ & $\begin{array}{l}\text { Prácticas } \\
\text { de Manejo }\end{array}$ & Vegetación \\
\hline JARDín & $1-4,6,7,10-13,15-30$ & $\begin{array}{c}\text { Comunes, injertadas } \\
\text { y de injerto }\end{array}$ & Protección & $\begin{array}{l}\text { Variable. Arbóreas, } \\
\text { arbustivas y herbáceas }\end{array}$ \\
\hline HUERTA & $1,6,10,15,21-24,30$ & Comunes y de injerto & Protección & $\begin{array}{l}\text { Herbáceas cultivadas } \\
\text { y pocas arbustivas }\end{array}$ \\
\hline MONTE FRUTAL & $\begin{array}{c}2-4,6,7,10-12 \\
15-24,26-28\end{array}$ & $\begin{array}{c}\text { Comunes, injertadas } \\
\text { y de injerto }\end{array}$ & $\begin{array}{l}\text { Tolerancia, } \\
\text { inducción y } \\
\text { protección }\end{array}$ & $\begin{array}{c}\text { Arbóreas y arbustivas, } \\
\text { también algunas } \\
\text { herbáceas }\end{array}$ \\
\hline Rozado & $\begin{array}{c}2,3,6,7,10,15- \\
17,21,24,27\end{array}$ & $\begin{array}{c}\text { Comunes, injertados } \\
\text { y de injerto }\end{array}$ & $\begin{array}{l}\text { Recolección, } \\
\text { tolerancia e } \\
\text { inducción }\end{array}$ & $\begin{array}{l}\text { Cultivos anuales rotativos } \\
\text { inmersos en el monte }\end{array}$ \\
\hline CAMPO & $\begin{array}{c}2,6,7,10,15,17 \\
18,21,24,30\end{array}$ & $\begin{array}{c}\text { Comunes, injertadas } \\
\text { y de injerto }\end{array}$ & $\begin{array}{l}\text { Recolección, } \\
\text { tolerancia e } \\
\text { inducción }\end{array}$ & $\begin{array}{l}\text { Herbáceas y arbustivas, } \\
\text { arbóreas ocasionales }\end{array}$ \\
\hline CAPUERA & $\begin{array}{c}2,5,6-8-10,14,15 \\
17,18,21,24,27\end{array}$ & Comunes y de injerto & $\begin{array}{l}\text { Recolección, } \\
\text { tolerancia e } \\
\text { inducción }\end{array}$ & Muy variable \\
\hline MONTE & $2,5,8,9,14,21,25,27$ & Comunes & Recolección & Arbóreas y arbustivas \\
\hline
\end{tabular}


estos espacios multiespecíficos son comunes los cítricos (tabla 2) y otros frutales introducidos como "durazno" [Prunus persica (L.) Batsch, Rosacae], "mango" (Mangifera indica L., Anacardiaceae), "kaqui" (Diospyros kaki L.f., Ebenaceae), "pecán" [Carya illinoinensis (Wangenh.) K.Koch, Juglandaceae], "castaña" (Castanea sativa Mill., Fagaceae), "vid" (Vitis vinifera L., Vitaceae), y frutales nativos como "pitanga" (Eugenia uniflora L., Myrtaceae), "araticú" (Rollinia emarginata Schltdl., Annonaceae), "guayaba" (Psidium guajaba L., Myrtaceae), "mamón" (Carica papaya L., Caricaceae) y "palta" (Persea americana Mill., Lauraceae). La disposición espacial puede ser azarosa u ordenada en líneas y surcos de distancia variable; disposición que cambia según las especies presentes, su procedencia y las costumbres familiares. Asimismo, el origen de los frutales incluidos en los mismos es tan variable como en los "jardines" antes descriptos, pudiendo ser por implantación o por tolerancia, más comúnmente ambos. En el primer caso se procede a la erradicación de la vegetación y posterior implantación de los frutales (Figura 2A, B), mientras que en el segundo, durante las tareas de "limpieza" del terreno, varias plantas preexistentes al microambiente son toleradas, sin haber sido plantadas (Figura 2D).

\section{HUERTA}

La "huerta" o "quinta" es un espacio cercano a la vivienda delimitado por un cerco perimetral (Figura 2C). Allí se cultivan diversas variedades de hortalizas $\mathrm{y}$ verduras, plantas medicinales, condimentos $\mathrm{y}$
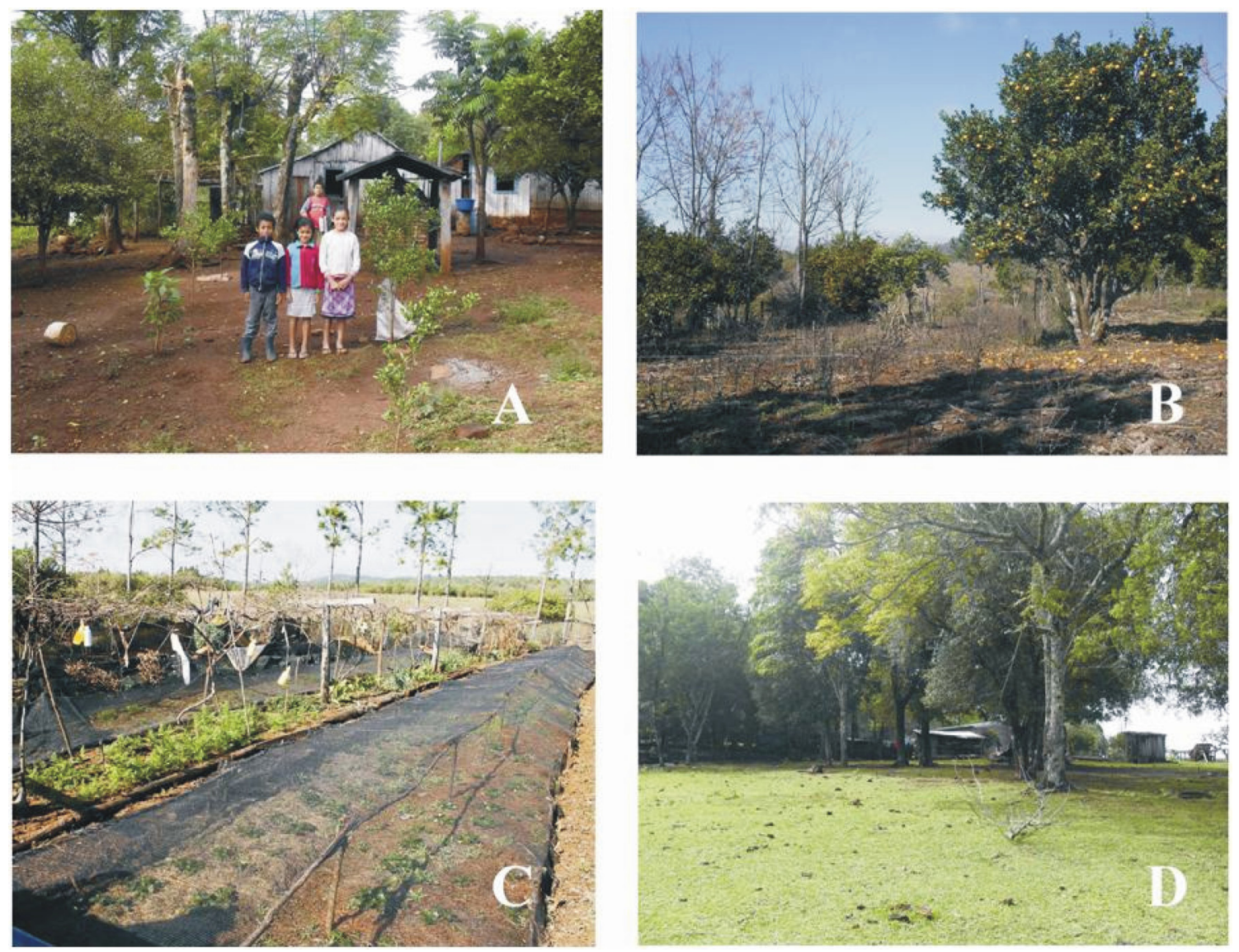

Fig. 2. Microambientes domésticos. A. Monte frutal recién implantado entre remanentes de uno antiguo. B. Monte frutal de cítricos. C. Huerta. D. Monte frutal por tolerancia. 


\section{P. C. Stampella - Domesticación del paisaje en Misiones (Argentina)}

frutales pequeños como "frutilla" [Fragaria $\times$ ananassa (Duchesne ex Weston) Duchesne ex Rozier, Rosacae] y "grosella" (Hibiscus sabdariffa L., Malvaceae). Cuando las dimensiones lo permiten este espacio es empleado como vivero para la siembra de propágulos de "ciruela" (Prunus domestica L., Rosaceae), "durazno" y cítricos.

\section{ROZADO}

El "rozado" o "labora" es una zona desmontada destinada a los cultivos anuales sin riego controlado. Frecuentemente, cada UD tiene varios "rozados" en diferentes estadios sucesionales como también varios de ellos activos a la vez en diferentes zonas, por lo cual las asociaciones se hacen muy diversas. Un mismo "rozado" puede tener diferentes parches caracterizados por distintos monocultivos asociados a características micro-locales. Por ejemplo, las plantaciones de "caña dulce" se asocian a los afloramientos rocosos, donde son más productivas (Figura 3A, B). Asimismo se observó en estos espacios algunas especies perennes, como el "ananá" o "abacayl" [Ananas comosus (L.) Merr., Bromeliaceae] y plantas arbóreas como cítricos, "níspero" [Eriobotrya japonica (Thunb.) Lindl., Rosacae], "palta", "mango", "yerba mate" y frutales del "monte", tolerados o plantados durante la apertura del "rozado".

$\mathrm{Su}$ superficie varía de unos pocos metros cuadrados hasta cerca de 5 hectáreas, dependiendo de la cantidad de miembros de la UD. Para su preparación se cortan los árboles y arbustos con machete y hacha (o en ocasiones con motosierras), se quema la vegetación restante y luego se labra la tierra con arado de tipo Romano tirado por bueyes (Figura 3C). Hasta hace pocos años, los "rozados"
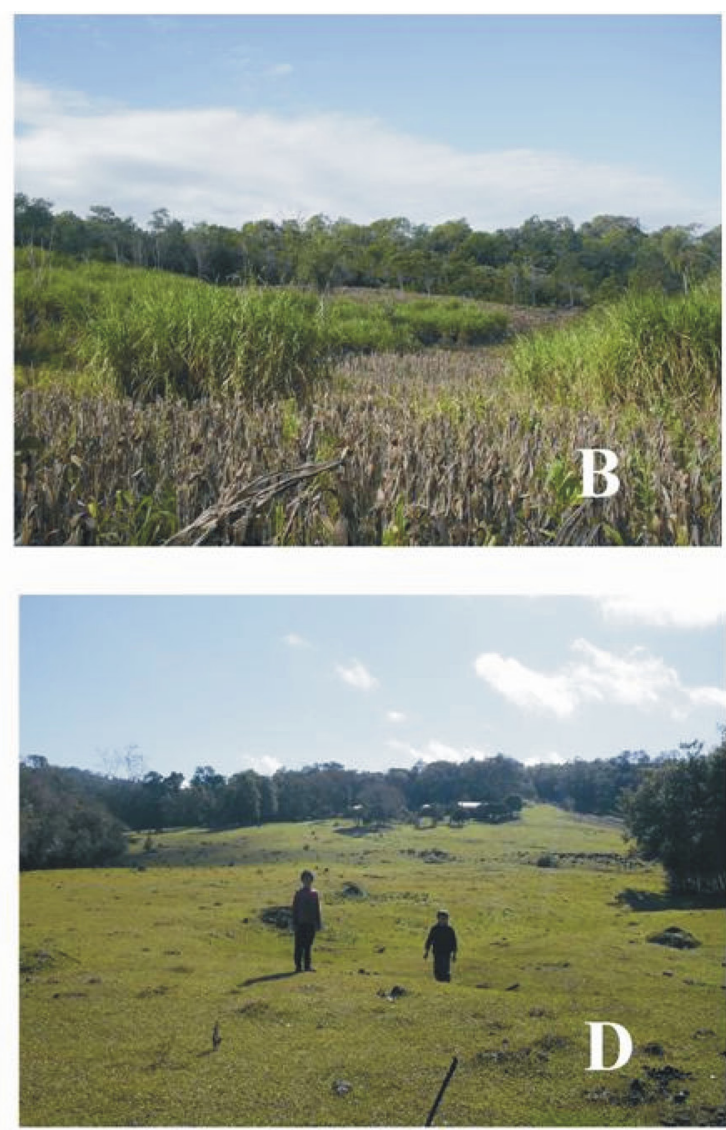

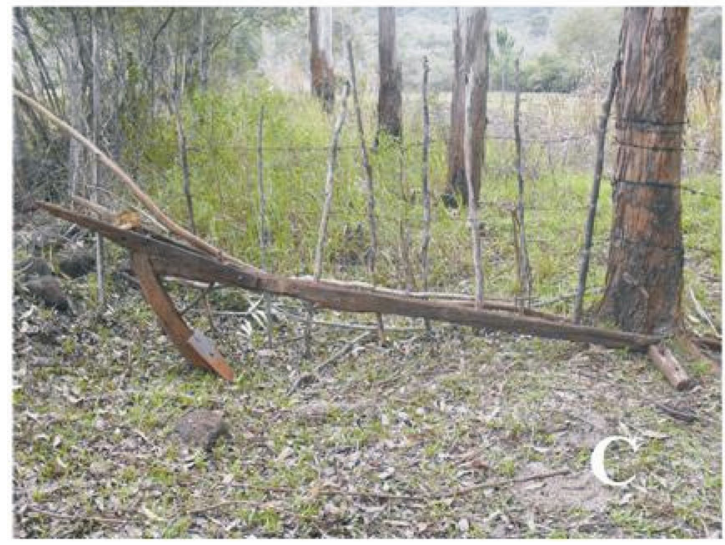

Fig. 3. Microambientes productivos. A. Rozado de mandioca, maíz y caña dulce. B. Rozado de maíz y caña dulce. C. Arado de tipo Romano empleado en las tareas de labranza. D. Campo peridoméstico. 
eran emplazados en zonas de "monte" talado y quemado para dicho fin, pero ahora acorde a las reglamentaciones de la Ley 26.331 (Protección de los Bosques Nativos) es frecuente la reutilización de las "capueras", es decir viejos "rozados" dejados en descanso varios años y en los que se regeneró parcialmente la vegetación leñosa. En este contexto, los "rozados" establecidos en sitios con suelos profundos se rotan cada 3 ó 4 años. En algunos enclaves de Teyú Cuaré, sitio con suelos más arenosos y pedregosos que en el resto de la región, luego de la tala no se practica la quema, antes del cultivo.

\section{CAMPO}

Es aquél ambiente donde predominan las plantas herbáceas. En la zona se establece en sitios con suelos poco profundos, sobre afloramientos rocosos o "pedreras" (Figura 3D). Se reserva la palabra "potrero" para el "campo" destinado a la cría del ganado, pudiendo estar inmerso dentro del "monte". Este espacio para el rodeo, pocas veces está delimitado por un alambrado u otro tipo de cercado. Rara vez se encuentran frutales plantados en los "campos", a excepción de aquellos cercanos a la vivienda donde pueden cuidarse las plántulas durante el crecimiento. Por esto, la mayoría de los frutales del "campo" tienen su origen en el avance de este ambiente sobre el "monte" o las "capueras", por lo cual, los cítricos presentes en este microambiente pertenecen a variedades "comunes". Las variedades injertadas que se hallaron corresponden a remanentes de antiguos "jardines" o "montes frutales". Entre los frutales se pueden mencionar: cítricos, "araticú", "huvenia" (Hovenia dulcis Thunb., Rhamnaceae), "guabirova" [Campomanesia xanthocarpa (Mart.) O. Berg, Myrtaceae], "soita" (Luehea divaricata Mart., Tiliaceae), "arasá" (Psidium sp., Myrtaceae), "pindó" [Syagrus romanzoffiana (Cham.) Glassman, Arecaceae], "coco" [Acrocomia aculeata (Jacq.) Lodd. ex Mart., Arecaceae], y en los sitios rocosos, "ananá", "caraguatá" (Bromelia sp., Bromeliaceae), "pitanga", "cerella" (Eugenia involucrata DC., Myrtaceae) y "talera” (Celtis sp., Celtidaceae).

\section{CAPUERAS}

Son ambientes productivos abandonados o en descanso, por ejemplo, un "jardín" abandonado, un "campo" sin ganado, un "rozado" no cultivado. Por esto el término es polisémico al referirse tanto a ambientes herbáceos, como arbustivos o arbóreos bajos, e incluso sucesiones más avanzadas similares al "monte" con elementos arbóreos de mayor diámetro. De acuerdo a las plantas que las componen, el grosor de los tallos y el estadío sucesional, este microambiente es denominado localmente "capuerita" (herbáceas o subarbustivas), "capuera fina" (arbustivas o arbóreas bajas) y "capuera gruesa" (arbóreas) (Figura 4AC). También toman el nombre de las especies dominantes: "chilcal" con dominancia de "chilca" (Baccharis sp., Asteraceae),"guaimirinsal" de "guaimirín" [Myrcia selloi (Spreng.) N. Silveira, Myrtaceae].

Los "capuerones" se parecen al "monte" pero poseen plantas arbustivas y subarbustivas en el estrato bajo. Son estimados para las excursiones de caza y recolección de frutas debido a la abundancia de frutales de varias especies, que atraen animales frugívoros. Esta abundancia se debe a la luminosidad, a la presencia de frutales remanentes de antiguos "montes frutales" y "jardines" y a la facilidad para el establecimiento y viabilidad de las "muditas". Ejemplos de estos frutales son cítricos y "palta" y, en los más antiguos se encuentran "agual" [Chrysophyllum marginatum (Hook. \& Arn.) Radlk., Sapotaceae], "jacaratiá" [Jacaratia spinosa (Aubl.) A.DC., Caricaceae], "ombu'" (Phytolacca dioica L., Phytolaccaceae) e "higuerón” [Ficus luschnathiana (Miq.) Miq., Moraceae].

\section{Monte}

Si bien el "monte" (o "matto") se trata al final por referencia a su distancia de la UD, en realidad constituye el espacio dominante en ambos enclaves y el que dio lugar -a través de su modificación- a los distintos microambientes referidos. Algunos "montes" poco intervenidos se consideran "vírgenes", tales como las selvas del Parque Provincial Teyú Cuaré y de Cerro Mártires (Figura 4D).

Este espacio es visto de diferentes maneras de acuerdo a la afiliación familiar. En general, los "colonos" y productores lo perciben como fuente de "maderas de valor" para la venta, para cría de ganado y para establecer cultivos. Para estas familias, el "monte" debe, necesariamente, 

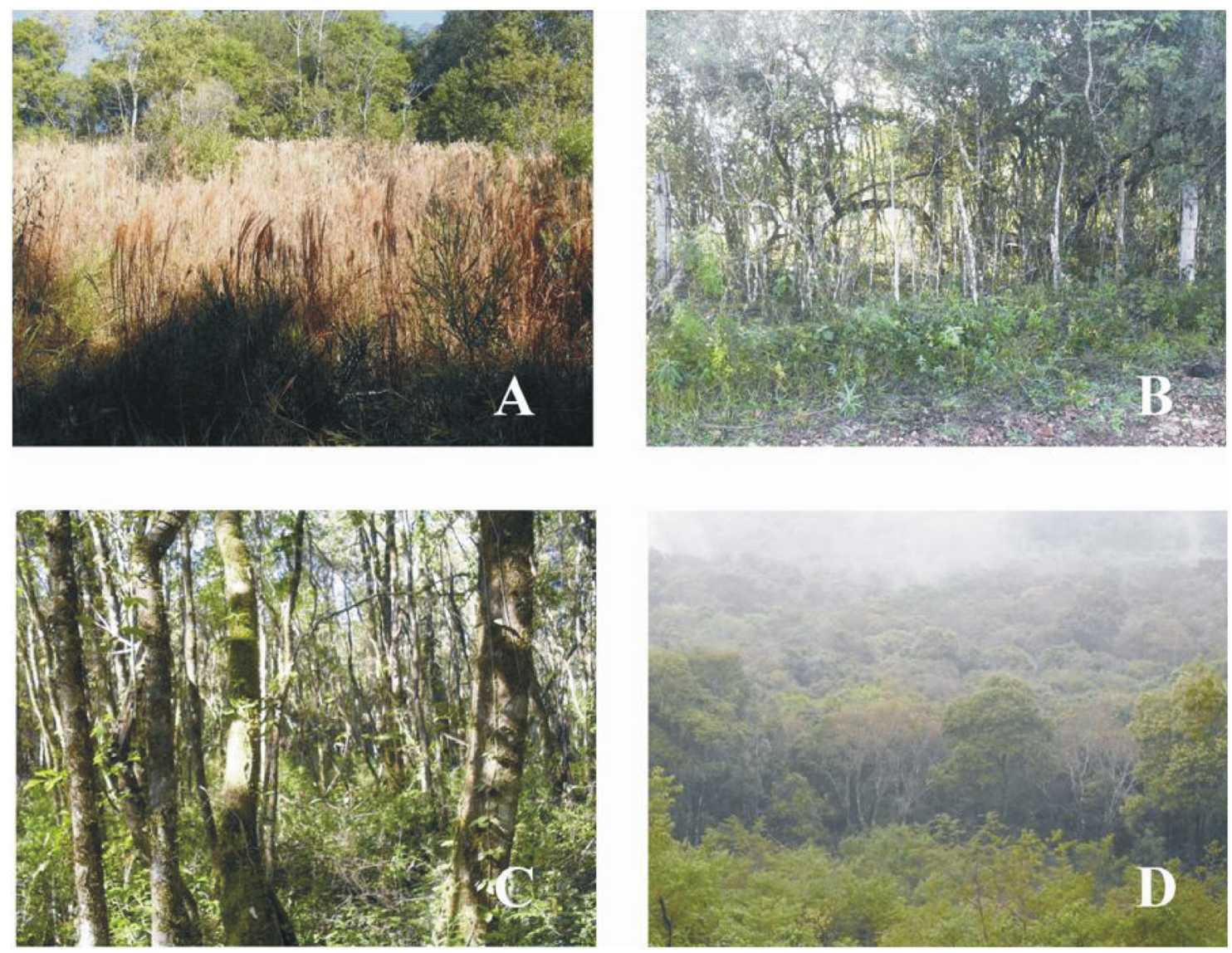

Fig. 4. Microambientes con bajo manejo local. A. Capuerita. B. Capuera fina. C. Capuera gruesa. D. Monte.

permanecer alejado del ámbito doméstico. No obstante, para los "criollos" -grupo más numeroso en la zona-, el "monte" cuenta con un significado más amplio, en especial si es peridoméstico, siendo considerado un bien común más de la UD, donde se llevan a cabo numerosas actividades cotidianas, inclusive el juego de los niños. Localmente se reconocen el "monte bajo" y el "monte alto". El primero se desarrolla sobre o en cercanías de las "pedreras" (Distrito del Urunday), mientras que el segundo está asociado a suelos profundos, ricos en materia orgánica.

\section{Prácticas de manejo}

Las prácticas de manejo relevadas se pueden sintetizar en dos niveles, aquellas focalizadas en las plantas propiamente dichas, y aquellas realizadas en cada uno de los microambientes particulares o grupos de microambientes. Por ejemplo, la poda es realizada sobre cada una de las plantas, mientras que la apertura de un nuevo "rozado" -con las actividades relacionadas de erradicación $\mathrm{y}$ tolerancia- tiene una incidencia mayor sobre las comunidades de plantas y animales.

\section{Prácticas sobre las plantas}

Al iniciarse el "monte frutal" y el "jardín", se emplean "muditas" espontáneas de otras viviendas y/o del "rozado", "campo", "capuera" o "monte". Cuando se desea cultivar alguna variedad particular, se siembran las semillas en almácigos, o directamente sobre el suelo tanto en el "rozado" como en los alrededores de la vivienda. Se utiliza para ello, con frecuencia, tierra extraída del "monte". Una vez establecidos los plantines, suelen ser protegidos de las bajas temperaturas y 
heladas con ramas de "guaimirín", ya que luego de cortadas, conservan las hojas un tiempo prolongado. En general, muy pocas prácticas se realizan sobre las plantas ya establecidas. Debido a la amplia disponibilidad de "muditas" en la zona, cuando una planta deja de producir es reemplazada por otra que ya se ha trasplantado en las cercanías. Las prácticas sobre las plantas de cítricos son podas, removido y abonado del suelo, reposición del suelo erosionado por escorrentía superficial, erradicación de malezas y enredaderas. En efecto, se podan eventualmente las ramas "chuponas" (aquellas de crecimiento vigoroso, espinosas y poco productivas); se realizan podas de rejuvenecimiento en el caso de plantas añosas; carpidas alrededor del tronco para eliminar principalmente la "grama" (gramíneas cespitosas) y aflojar la tierra; abonado con ramas trituradas y bosta de vaca (Figura 5A). En suelos con pendiente se nivela con piedras siguiendo las curvas de nivel y se rellena con tierra en el caso de haberse erosionado por escorrentía superficial (Figura 5B). Asimismo, se eliminan las "sipó" (enredaderas y trepadoras) que cubren los frutales. En el caso del "monte frutal" los abonos y el control de malezas son realizados por los animales de granja, a los que se les permite transitar casi todos los espacios domésticos, a excepción del "jardin" y la "huerta".

Las técnicas de cosecha de los frutos varían de acuerdo al porte de la planta. En plantas de grandes dimensiones ("comunes") se emplea un "gancho" que consiste en cualquier rama cortada con machete, prefiriéndose aquellas de "talera" debido al profuso patrón de ramificación de las mismas (Figura 5C). En este caso los frutos lejanos son alcanzados con el "gancho" tirando hacia abajo para desprenderlos. Otra variante es la "horqueta", rama larga que remata en una dicotomía, empleada para agarrar frutos o grupos de frutos mientras se enrosca la "horqueta" hacia un lado hasta cortar las ramas fructíferas (Figura 5D). Durante el período de fructificación de las plantas es común encontrar estos implementos apoyados en las mismas para quien quiera alcanzar los frutos. Otro modo de cosechar en plantas más altas ("de monte") es trepando a la misma, práctica realizada por los niños que ascienden a la planta y desde allí arrojan las frutas al piso. En ambos enclaves, se ha relevado la tala del ejemplar (erradicación) con el objeto de acceder a los frutos más altos, siendo común en los relatos locales.

\section{Prácticas sobre los microambientes}

Las prácticas de manejo que inciden en la distribución, abundancia y calidad de fruta de los cítricos -como de otros frutales- sin dudas son los factores antrópicos más importantes en el modelado de este recurso local. En primer lugar, se observa la recolección y consumo in situ de frutos durante las actividades cotidianas. El desplazamiento por los "trillos" (senderos) hacia los distintos microambientes casi siempre va acompañado del consumo de frutas. Si los "trillos" se encuentran en el "monte" o en la "capuera" las semillas usualmente germinan, especialmente en aquellos ambientes donde hay un manejo (raleo) de la cobertura arbórea. Esto no ocurre en el "campo" debido a la presencia de la "grama". Del mismo modo el ganado es un agente dispersor asociado a la actividad humana. Dado que los "potreros" generalmente no poseen delimitaciones permanentes, si los "montes" y "capueras" que lo circundan cuentan con plantas de estos frutales, se vuelven sitios de forrajeo intenso durante la fructificación, especialmente durante el invierno cuando los cítricos fructifican y escasean las pasturas. Como consecuencia, en ambos casos, se observa una mayor concentración de renovales y ejemplares adultos de cítricos en los bordes del "monte" y en los "trillos". El consumo doméstico a la vez promueve la relocalización de las distintas etnoespecies, de ese modo los frutos "de monte" son trasladados como "muditas" desde el "monte" al hogar.

Como ya se ha referido anteriormente en los microambientes ("rozado", "campo", "jardín" y "monte frutal") los frutales son tolerados durante las tareas de tala del "monte" o "capuera". De ese modo, y de acuerdo a distintos criterios de preferencia, los cítricos "de monte" son incorporados a la UD, donde pasan a ser denominados "caseros". Generalmente, estos frutales adquieren características deseables por el mero hecho de pertenecer a los microambientes domésticos: "cargan más" (mayor productividad), con fruta más "graúda" (grandes), jugosas y dulces; así como también disminuye el tiempo de maduración, y el porte de la planta y la ramificación se torna más densa y achaparrada, facilitando así la recolección de los frutos. En la concepción local el "monte" les quita dulzor y jugosidad a las plantas, por lo tanto al alejarse los frutales del "monte" éstos cambian: "Hay algunas [naranjas] 

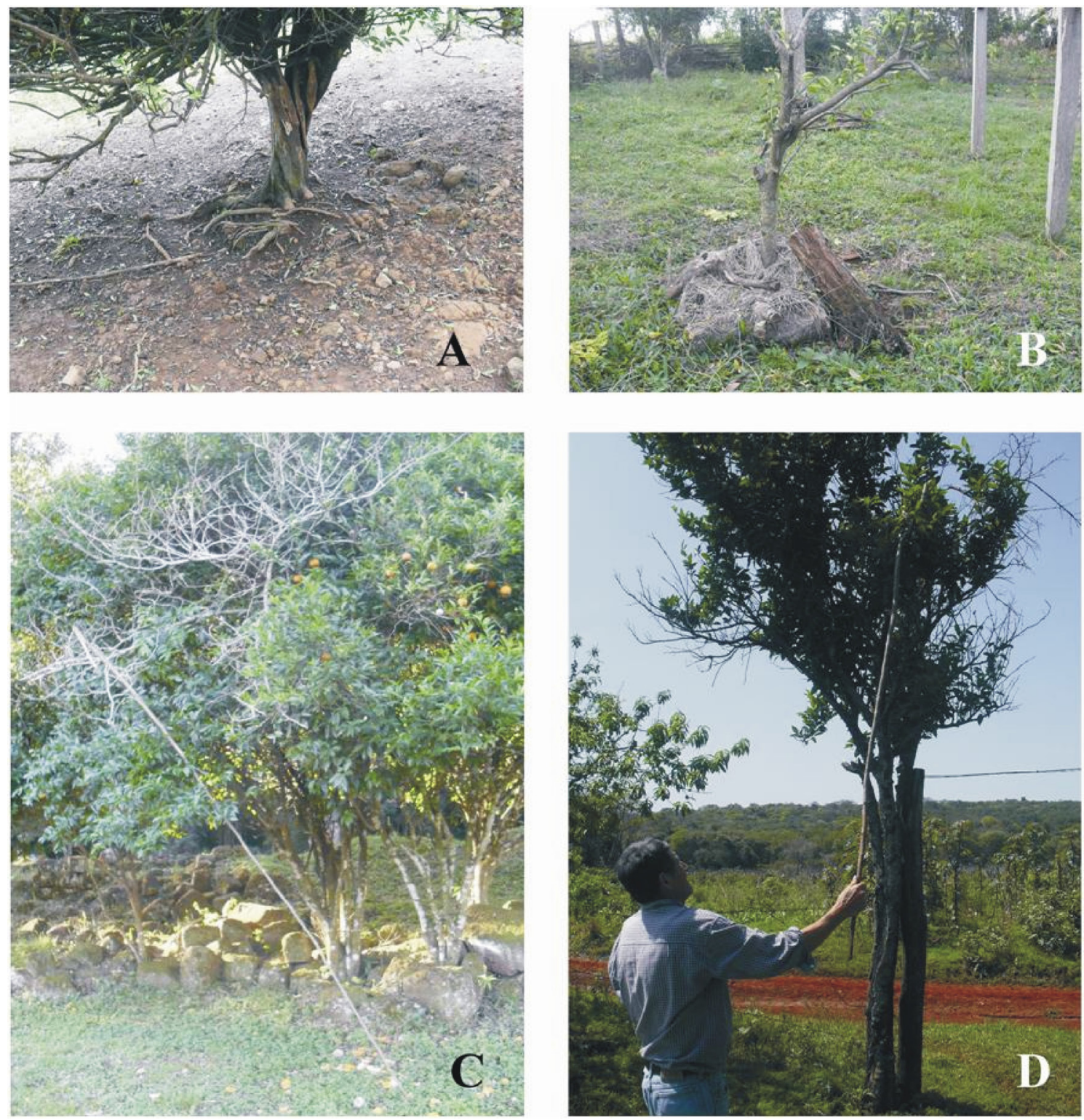

Fig. 5. Prácticas de manejo sobre las plantas y herramientas de recolección de frutos. A. Erosión del suelo. B. Abonado y reposición del suelo erosionado. C. Gancho. D. Horqueta.

que son más simples, que tienen menos dulzura porque seguramente los árboles del monte le sacan la dulzura, sí eso les pasa. Y hay alguna que está medio salida del monte, en el costado, que son bien dulce" (S.F., 65 años, Cerro Mártires).

Una vez incluidas en el nuevo microambientes las plantas toleradas pueden ser fomentadas y protegidas, de acuerdo a las prácticas nombradas para las plantas del "jardín" y "huerta". Las prácticas de fomento incluyen actividades simples y directas como el consumo in situ de los frutos y dispersión de sus semillas, la eliminación de plantas no deseadas que crecen en las cercanías y la ocasional siembra de propágulos; así como actividades indirectas, tales como la cría de ganado en el "monte", especialmente durante el período invernal. 
Estas prácticas configuran flujos de germoplasma entre los distintos microambientes donde intervienen también el "encapuerado" (regeneración natural). El complejo conformado por el "jardín", "huerta" y "monte frutal", es receptor de plántulas y semillas al iniciar nuevas plantaciones, por consumo de frutos "de monte" en los alrededores del hogar y por las deposiciones de las aves. El "rozado", generalmente alejado espacialmente de los demás microambientes e inmerso en el "monte", está relacionado con este último. Si bien algunos frutales del "jardín" o "monte frutal" son plantados allí, la mayoría provienen de la tolerancia de los frutales "de monte" en el avance de este microambiente y por establecimiento de plantas espontáneas. En el "campo", en cambio, debido a la cubierta de gramíneas y a la actividad de forrajeo y pisoteo del ganado, el establecimiento de plántulas provenientes de semillas es infrecuente, derivando casi exclusivamente los frutales de la tolerancia ante el avance sobre el "monte". Finalmente, el "monte" es receptor de germoplasma por escape de cultivo de las plantas "caseras" y por "encapuerado" al avanzar el "monte" sobre los demás microambientes.

Las prácticas de manejo en la dinámica entre el monte y el campo

Estos microambientes descriptos no se mantienen estáticos. Según las prácticas locales la frecuencia y extensión de éstos varía. Como ya se refirió para el "monte", las apreciaciones de los distintos microambientes por los diferentes grupos ("paisanos", "criollos", "colonos" y productores) no ocurren de la misma manera. Para los "paisanos", y en parte para los "criollos", la "capuera" anuncia la regeneración del "monte", microambiente preciado para las actividades de recolección y caza; mientras que para los "colonos" y productores indica desidia, ya que está relacionado con un espacio "sucio" e "improductivo", susceptible a ser transformado en un espacio productivo.

El "monte" es el microambiente prototípico de la zona, ya que todos los demás microambientes derivan de la tala del "monte" y tienden a "monte" previo paso por "capuera". En algunas zonas el "campo" ya se encuentra establecido y permanece debido a la pobreza del suelo y a las "pedreras" que afloran, muchas veces atribuidas a prácticas no sustentables y a antiguas viviendas, donde el suelo fue erosionado por escorrentía superficial durante las épocas lluviosas.
Los informantes recurren a prácticas de manejo sobre los "campos" que garantizan la permanencia de este microambiente. Al avanzar este microambiente sobre el "monte" o "capuera" mediante la roza y quema de los árboles y arbustos, se favorece el crecimiento de comunidades herbáceas, impidiendo el establecimiento de plántulas de especies leñosas. Sin embargo, varias plantas útiles que ya fueron mencionadas anteriormente son toleradas: "en el campo uno deja siempre por ahi una planta de mandarina o naranja o de guabiyú [Myrcianthes pungens (O. Berg) D. Legrand, Myrtaceae] o de pitanga (...) para que uno tenga para uno comer. (...) Casi todos son remedio, muchas cosas que tal vez uno cree que no es remedio y es remedio (S.F., 65 años, Cerro Mártires). El retiro de los animales del "campo" generalmente es seguido por la invasión de "mata campo" [Vernonanthura tweedieana (Baker) H.Rob, Asteraceae] que vuelve poco productivo este microambiente al disminuir la abundancia de pasturas. Al contrario de lo expuesto anteriormente el proceso inverso es menos costoso. La pregunta acerca de las prácticas que fomentan el avance del "monte" sobre el "campo" resultó graciosa a los informantes, respondiendo que "el monte avanza solito" o "sólo sacando la vaca nomás, pero ¿para qué...?". Entre las plantas pioneras en el avance del "monte" sobre el "campo" fueron mencionadas: "una planta de bacún [Allophylus edulis (A.St.Hil., A.Juss. \& A.Cambess.) Radlk., Sapindaceae], de pitanga, de aruera [Lithraea molleoides (Vell.) Engl., Anacardiaceae], de cualquier cosa. Y viene viniendo toda esa planta tomando cuenta el campo. Y cuando se da de cuenta, si es un campo medio chico, él toma cuenta, sí. Encapuera primero, de maderita fina primero, $y$ de a poco va engrosando y se pone viga o se pone un barrotón grueso" (F. B., 74 años, Cerro Mártires).

\section{Discusión}

En los cítricos puede apreciarse, en un mismo paisaje, un continuum de variedades representantes del proceso de domesticación y que varían desde poblaciones espontáneas en microambientes con baja intervención antrópica (etnovariedades " $d e$ monte"), pasando por aquellas incipientemente domesticadas ("caseras"), hacia las completamente 


\section{P. C. Stampella - Domesticación del paisaje en Misiones (Argentina)}

domesticadas (muchas de las variedades “injertadas"). En ese sentido, la diversidad local relevada por Stampella (2016) se ajusta a este gradiente coincidiendo, a su vez, con el complejo cultivo-maleza-domesticado propuesto por Beebe et al. (1997) para Phaseolus vulgaris L. (Fabaceae). En el mismo, se propone la persistencia de este complejo de variedades que se encuentran en estrecho contacto debido a las estrategias de cultivo, como también al empleo de prácticas de manejo que resulten en un paisaje incipientemente domesticado y en la tolerancia de variedades arvenses. Estas últimas, para el caso de los cítricos, refieren a aquellas que ingresan al "jardín", "monte frutal", "huerta" y "rozado", y que permanecen como espontáneas al ser toleradas. Varios cultivos han sido analizados desde esta perspectiva resaltando la importancia de las nuevas combinaciones generadas por hibridación de los mismos con las variedades "silvestres" y la importancia de la diversidad para los productores y para la resiliencia de sus sistemas productivos (Jarvis \& Hodgkin, 1999).

$\mathrm{El}$ ingreso de los frutales "de monte" a la UD, y su consiguiente cambio de nominación (Stampella, 2016), incrementa la diversidad de etnovariedades (tabla 2). No es que es realidad se trate de especies o variedades diferentes sino de las mismas variedades que, al crecer (o al ser cultivadas) en distintos microambientes varían sus atributos de manera cuali-cuantitativa. Sin embargo, los informantes las perciben como etnovariedades diferentes basándose en los cambios morfológicos-organolépticos como el tiempo de maduración, cantidad de jugo, acidez/ dulzura de la pulpa y altura de la planta de acuerdo al microambiente al cual están relacionadas, pudiendo interpretarse como una estrategia de diversificación a diferentes escalas, relacionada tanto a aspectos biológicos como socioculturales. Estas características morfofisiológicas seleccionadas inconscientemente, y relacionadas a los diferentes microambientes antropogénicos, son denominadas en su conjunto "síndrome de domesticación" (Gepts, 2004; Zohary, 2004) y han sido estudiadas en otros frutales arbóreos como Chrysophyllum cainito L. Estos trabajos han hallado diferencias significativas en el tamaño de los frutos, concentración de azúcares, proporción de endocarpo, cantidad de compuestos fenólicos y grosor del exocarpo, comparando poblaciones cultivadas y silvestres, siendo los dos primeros los más importantes en la diferenciación de las poblaciones (Parker et al., 2010). Lins Neto et al. (2012) no hallan diferencias morfofisiológicas significativas en poblaciones de $S$. tuberosa pertenecientes a diferentes microambientes. Sin embargo, los frutales pertenecientes a los ambientes antropogénicos presentan características más estimadas.

Así, desde un punto de vista sistemático, las etnovariedades "de monte" y "caseras" representan la misma variedad, sólo que una crece en el "monte" y la otra en la "casa". Este "movimiento", descripto para Solanum tuberosum L. (Solanaceae) y otros cultivos andinos por Lema (2013, 2014) está acompañado por cambios morfológicos y organolépticos, como también en la relación ser humano-planta.

Este mismo flujo ocurre en sentido inverso ("naturalización" o escape de cultivo) y representa el origen de algunos cítricos "de monte" como "naranja" y "apepú" a partir de plantas "caseras" durante el período jesuítico (siglos XVII-XVIII). Este flujo hacia el "monte" ha sido reportado en la zona para varios frutales cítricos como "apepu', "limón mandarina" y "mandarina común" (Gade, 1976; Stampella et al., 2013a, 2014), como también para otros frutales locales como "níspero", "pecán" y una especie de Butia (Arecaceae) (Krapovickas \& Dematteis, 2008; Delucchi \& Keller, 2010; Hurrell et al., 2011). Este proceso de "naturalización" pocas veces toma en cuenta prácticas de manejo actuales y pasadas que fomentan dicho proceso, por lo cual el componente humano queda invisibilizado. Es por eso que los trabajos citados anteriormente se han enfocado no sólo en la descripción del taxón novedoso para la flora y sus características ecológicas, sino también en su relación con las comunidades locales. Visto desde otra óptica, esto se ha denominado también "antropización" de las plantas, dado que las mismas no crecen en ambientes exentos de prácticas sino con manejo de pobladores locales muchas veces no tenidos en cuenta (Hilgert et al., 2014).

Sin embargo, la preferencia de "plantas semilleras" (pies productores de semillas) de familiares y vecinos (plantas "caseras") antes que "de monte" contribuye a la conservación de etnovariedades seleccionadas a lo largo del tiempo (Parra et al., 2010; Aguirre-Dugua et al., 2012). Esta práctica de sembrar variedades 
"de monte" y "caseras" implica hibridación y generación de variabilidad, y luego selección de la diversidad antes que conservación ex situ contribuyendo a la conservación dinámica del complejo cultivo-maleza-domesticado. Las prácticas de manejo se complejizan más aún al tener en cuenta la versatilidad reproductiva de este género que involucra procesos de hibridación inter e intraespecífica, apomixis y poliploidía conocidos como "enjambre híbrido" (Grant, 1989). Por lo tanto, la propagación de etnovariedades particulares podría realizarse mediante injertos, aislamiento reproductivo (genético, geográfico) o autopolinización.

Las prácticas mencionadas para la implantación de los "montes frutales" son semejantes a las descriptas por Sartori (1964), difiriendo en los patrones de distancias entre líneas y ejemplares como también en la relación de las plantas con el sustrato, pero sí son novedosas las prácticas de manejo que involucran la tolerancia y fomento de frutales preexistentes. Así, en este espacio convergen frutales preexistentes e implantados conformando sistemas agroforestales diversos.

Por otra parte, existen referencias a prácticas lesivas como la tala en San Ignacio ya hacia fines del siglo XIX (Ambrosetti 2008: 87) y constituyen, para el imaginario local, una de las causas de desaparición de los "naranjales de monte" y de las ruinas jesuíticas. Sin embargo, es posible que la disminución de los "naranjales" esté más relacionada a cambios en los sistemas productivos regionales, a las prácticas de manejo sobre el "monte" y a la urbanización, antes que a prácticas de erradicación localizadas.

Las incursiones de caza y recolección a los "capuerones" antes que al "monte" están relacionadas a intervenciones antrópicas del ambiente de acuerdo a prácticas sustentables enmarcadas en la EUM (Toledo \& Bassols, 2008). De esta manera, la diversidad de plantas útiles se incrementa en microambientes relacionados a este tipo de prácticas como lo demuestra Balée (1993) para el Amazonas brasileño donde algunos parches de selva, derivados de antiguos "rozados", presentan valores de números de individuos, especies y familias, similares a los relevados en los parches de selva densa, pero conformados principalmente por especies útiles y que prosperan bajo manejo agroforestal.

\section{Conclusiones}

La diversidad de microambientes donde se cultivan o crecen espontáneamente los cítricos, genera a su vez diversidad morfológica, organoléptica y etnotaxonómica, al ser clasificada de acuerdo a percepciones locales. Todos los microambientes mencionados presentan prácticas de manejo para el establecimiento y continuidad de los mismos, como también para la diversificación y disponibilidad de las variedades particularmente. Estos microambientes pueden intercambiarse en el tiempo de acuerdo a las prácticas de manejo, permaneciendo algunas plantas toleradas en la nueva conformación. Así, lo que hoy percibimos como "monte" puede ser un antiguo "monte frutal" con plantas toleradas, que pueden percibirse como "naturalizadas", al desconocer su historia.

Este repertorio amplio de prácticas de manejo diversificadoras se encuentra enmarcado en una EUM a diferentes niveles que posibilita a los pobladores locales disponer de estos frutales a lo largo del ciclo anual, empleando variedades "comunes" y diferentes microambientes donde son cultivadas, casi sin emplear variedades injertadas.

\section{Agradecimientos}

A Emilia y Juana por el amor que hay detrás de este trabajo. A CONICET, UNLP y MINCyT, por el apoyo económico brindado. A mis directoras, M.L. Pochettino y N. Hilgert, que enriquecieron este trabajo a lo largo de todo el proceso, al igual que $\mathrm{J}$. Hurrell, G. Delucchi y H. Keller. A P. Cabanillas, R. Dutra, V. Furlán y M. Núñez por su apoyo en las campañas. A las comunidades locales, personal de las ruinas de Santa María la Mayor, y de la Escuela № 509 de Cerro Mártires, por compartir su tiempo, conocimientos y amistad.

\section{Bibliografía}

ALBUQUERQUE, U. P., L. V. DA CUNHA, R. F. DE LUCENA \& R. R. ALVES (eds.). 2014. Methods and techniques in ethnobiology and ethnoecology. Humana Press, New York.

ANDERSON, C. 1996. Variedades cultivadas en el área del Río Uruguay. In: FABIANI, A., R. MIKA, L. LAROCCA \& C. ANDERSON (eds.), Manual para 


\section{P. C. Stampella - Domesticación del paisaje en Misiones (Argentina)}

productores de naranja y mandarina de la región del Rio Uruguay, pp. 63-92. Diversificación Productiva, Manual Serie A Nro. 2. INTA, Concordia.

BELASTEGUI, H. M. 2006. Los colonos de Misiones. Editorial Universitaria de Misiones, Posadas.

BALÉE, W. 1993. Indigenous transformation of Amazonian forests: an example from Maranhão, Brazil. L'Homme 33: 231-254.

BALÉE, W. (ed.). 1998. Advances in Historical Ecology. Columbia University Press, New York.

BEEBE, S., O. TORO., A. V. GONZÁlEZ, M. I. CHACÓN \& D. G. DEBROUCK. 1997. Wildweed-crop complexes of common bean (Phaseolus vulgaris, Fabaceae) in the Andes of Peru and Colombia, and their implications for conservation and breeding. Genetic Resources and Crop Evolution 44: 73-91.

CASAS, A. 2001. Silvicultura y domesticación de plantas en Mesoamérica. En: AGUILAR, B. R., S. R. DOMÍNGUEZ, J. CABALlERO \& M. A. M. ALFARO (eds.), Plantas, cultura y sociedad. Estudio sobre la relación entre seres humanos y plantas en los albores del siglo XXI, pp. 123-157. Universidad Autónoma Metropolitana, México.

CASAS, A. \& J. CABALLERO. 1996. Traditional management and morphological variation in Leucaena esculenta (Fabaceae: Mimosoideae) in the Mixtec region of Guerrero, Mexico. Econ. Bot. 50: $167-181$.

DELUCCHI, G. \& H. A. KELLER. 2010. La naturalización del "níspero", Eriobotrya japonica (Rosaceae, Maloideae), en la Argentina. Bonplandia 19: 71-77.

DUGUA, X. A., L. E. EGUIARTE, A. G. RODRÍGUEZ \& A. CASAS. 2012. Round and large: morphological and genetic consequences of artificial selection on the gourd tree Crescentia cujete by the Maya of the Yucatan Peninsula, Mexico. Ann. Bot. 109: 12971306.

EMPERAIRE, L., F. PINTON \& G. SECOND. 2001. Dinámica y manejo de la diversidad de las variedades de yuca del noroccidente amazónico (Brasil). Etnoecológica 5: 38-59.

ETKIN, N. L. \& T. TICKTIN. 2010. Advancing an ethnoecological perspective that integrates theory and method in ethnobotany. In: ALBUQUERQUE, U. P. \& N. HANAZAKI (eds.), Recent developments and case studies in ethnobotany, pp. 33-57. NUPEEA, Recife.

GADE, D. W. 1976. Naturalization of plant aliens: the volunteer orange in Paraguay. J. Biogeogr. 3: 269279.

GEPTS, P. 2004. Crop domestication as a long-term selection experiment. Plant Breeding Reviews 24: $1-44$.
GONZÁLEZ-SOBERANIS, M. C. \& A. CASAS. 2004. Traditional management and domestication of tempesquistle, Sideroxylon palmeri (Sapotaceae) in the Tahuacán Valley, Central Mexico. Journal of Arid Environments 59: 245-258.

GRANT, V. 1989. Especiación Vegetal. Ed. LIMUSA S.A., Mexico.

GUARINO, L. \& M. HOOGENDIJK. 2004. Microenvironments. In: EYZAGUIRRE, P. B. \& O. F. LINARES (eds.), Homegardens and Agrobiodiversity, pp. 31-40. Smithsonian Institution, Washington.

HARLAN, J. R. 1992. Crops \& Man. American Society of Agronomy, Inc. Madison.

HILGERT, N. I., D. A. LAMBARÉ, N. D. VIGNALE, P. C. STAMPELLA \& M. L. POCHETTINO. 2014. ¿Especies naturalizadas o antropizadas? Apropiación local y la construcción de saberes sobre los frutales introducidos en época histórica en el norte de Argentina. Revista Biodiversidad Neotropical 4: 69-87.

HURRELL, J. A., G. DELUCCHI \& H. A. KELLER. 2011. Carya illinoensis (Juglandaceae) adventicia en la Argentina. Bonplandia 20: 47-54.

JARVIS, D. \& T. HODGKIN. 1999. Wild relatives and crop cultivars: detecting natural introgression and farmer selection of new genetic combinations in agroecosystems. Molecular Ecology 8: 159-173.

KRAPOVICKAS, A. \& M. DEMATTEIS. 2008. Butia eriospatha, palmera naturalizada en el norte de Misiones (Argentina). Bonplandia 17: 91-92.

LEMA, V. S. 2013. Crianza mutua: una gramática de la sociabilidad andina. In: CABALLERO, I. V. \& F. PAZZARELLI (coord.), Entre Andes y Amazonía: transformaciones de materias, sustancias y cuerpos. Actas de la X Reunión de Antropología del Mercosur. 10 al 13 de julio, Córdoba.

LEMA, V. S. 2014. Hacia una cartografía de la crianza: domesticidad y domesticación en comunidades andinas. Espaço Amerindio 8: 59-82.

NETO, E. M., N. PERONI, C. M. MARANHÃO, M. I. MACIEL \& U. P. ALBUQUERQUE. 2012. Analysis of umbu (Spondias tuberosa (Anacardiaceae)) in different landscape management regimes. Environmental Monitoring and Assessment 184: 4489-4499.

MARIUS, R. M., B. SINSIN, O. EYOG-MATIG \& R. FINKELDEY. 2010. Uses, traditional management, perception of variation and preferences in ackee (Blighia sapida) fruit traits in Benin: implications for domestication and conservation. J. Ethnobiol. Ethnomed. 6: 12.

MARTIN, G. J. 2001. Etnobotánica, Manual de Métodos. Ed. Nordan-Comunidad, Montevideo.

MILLER, A. J. \& B. L. GROSS. 2011. From forest to field: perennial fruit crop domestication. Amer. $J$. Bot. 98: 1389-1414. 
PALACIOS, J. 1978. Citricultura Moderna. Ed. Hemisferio Sur, Buenos Aires.

PARKER, I. M., I. LÓPEZ, J. J. PETERSEN, N. ANAYA, L. C. RÍOS \& D. POTTER. 2010. Domestication syndrome in Caimito (Chrysophyllum cainito): fruit and seed characteristics. Econ. Bot. 64: 161-175.

PARRA, F.,A.CASAS, J. M. RAMÍREZ,A.C.PALOMEC, V. R. RAMÍREZ \& A. G. RODRÍGUEZ. 2010. Evolution under domestication: ongoing artificial selection and divergence of wild and managed Stenocereus pruinosus (Cactaceae) populations in the Tehuacán Valley, Mexico. Ann. Bot. 106: 483-496.

POCHETTINO, M. L., D. A. LAMBARÉ, P. STAMPELLA, M. B. DOUMECQ \& N. GHIANIECHENIQUE. 2015. Especies arbóreas como "texto" en contextos pluriculturales... La conservación como pretexto [on line]. XI Reunión de Antropología del Mercosur. Montevideo. Disponible en: http://http:// xiram.com.uy/actas-del-congreso/grupos-de-trabajo/ ponencias-grupo-de-trabajo-83 [Acceso: 20 de enero de 2017].

RIAT, P.; P. C. STAMPELLA \& M. L. POCHETTINO. Incidencia de la estrategia de uso múltiple en la autosubsistencia de dos comunidades campesinas de la Argentina. GAIA Scientia, en prensa.

SARTORI, E. 1964. Árboles frutales. En: PARODI, L. R. (ed.), Enciclopedia Argentina de Agricultura y Jardinería. El cultivo de las plantas útiles, pp. 789927. Volumen 2, parte 2. Ed. ACME S.A., Buenos Aires.

SCHADEN, E. 1998. Aspectos fundamentales de la cultura Guarani. CEADUC: Centro de Estudios Antropológicos, Universidad Católica N.S. de la Asunción, Asunción.

STAMPELLA, P. C. 2015. Historia local de naranja amarga (Citrus $\times$ aurantium, Rutaceae) del Viejo Mundo asilvestrada en el corredor de las antiguas Misiones Jesuíticas de la provincia de Misiones (Argentina). Caracterización desde una perspectiva interdisciplinaria. Tesis Doctoral, Biblioteca de la FCNyM, UNLP.

STAMPELLA, P. C. 2016. Local variability of citrus (Citrus spp.) in pluricultural enclaves in the south of Misiones (Argentina): Diversity, uses and perception, and classification of variability. J. Ethnobiol. 36: 637-657.

STAMPELLA, P. C., G. DELUCCHI \& M. L. POCHETTINO. 2013a. Naturalización e identidad del "limón mandarina", Citrus $\times$ taitensis (Rutaceae, Aurantioideae) en la Argentina. Bol. Soc. Argent. Bot. 48: 161-169.
STAMPELLA, P. C., N. I. HILGERT \& M. L. POCHETTINO. Usos medicinales de los cítricos (Citrus L., Rutaceae) entre los criollos del sur de Misiones (Argentina). GAIA Scientia, en prensa.

STAMPELLA, P. C., D. A. LAMBARÉ, N. I. HILGERT \& M. L. POCHETTINO. 2013b. What the iberic conquest bequeated to us: the fruit trees introduced in argentine subtropic, their history and importance in present traditional medicine. eCAM. Article ID 868394, 17 pages.

STAMPELLA, P. C., H. A. KELLER, J. A. HURRELL \& G. DELUCCHI. 2014. Etnobotánica y naturalización de dos cultivares de Citrus reticulata en la Argentina. Bonplandia 23: 151-162.

SWINGLE, W. T. 1943. The botany of citrus and its wild relatives of the orange subfamily. In: WEBBER, H. J. \& L. D. BATCHELOR (eds.), The Citrus Industry. History, Botany, and Breeding, pp. 129474. University of California Press, Berkeley.

THE PLANT LIST. 2016. A working list of all plant species [online]. Disponible en: http://www. theplantlist.org [acceso: 26 de octubre 2016].

TOLEDO, V. \& N. B. BASSOLS. 2008. La memoria biocultural. La importancia agroecológica de las sabidurías tradicionales. Icaria Editorial, Barcelona.

TOLEDO, V. M., N. B. BASSOLS, E. G. FRAPOLLI \& P. A. CHAIRES. 2007. Manejo y uso de la biodiversidad entre los Mayas Yucatecos. Biodiversitas 70: 10-15.

VODOUHÈ, R. \& A. DANSI. 2012. The "bringing into cultivation" phase of the plant domestication process and its contributions to in situ conservation of genetic resources in Benin. The Scientific World Journal 2012, Article ID 176939, 13 pages.

WEBBER, H. J. 1943. Cultivated varieties of Citrus. In: WEBBER, H. J. \& L. D. BATCHELOR (eds.), The Citrus Industry. History, Botany, and Breeding, pp. 475-668. University of California Press, Berkeley.

ZOHARY, D. 2004. Unconscious selection and the evolution of domesticated plants. Econ. Bot. 58: $5-10$.

Recibido el 29 de mayo de 2017, aceptado el 24 de julio de 2017. Editor: Norma Hilgert. 